\title{
Síndrome de Tolosa-Hunt: una causa infrecuente de oftalmoplejía
}

\author{
Juan Cantón-De-Seoane, Gema Flox-Benítez, Blanca Alonso-Martínez \\ Servicio de Medicina Interna. Hospital Severo Ochoa. Leganés. Madrid. España
}

Recibido: 17/09/2020

Aceptado: 10/11/2020

En línea: 31/12/2020

Citar como: Cantón-De-Seoane J, Flox-Benítez G, Alonso-Martínez B. Síndrome de Tolosa-Hunt: una causa infrecuente de oftalmoplejía. Rev Esp Casos Clin Med Intern (RECCMI). 2020 (Dic); 5(3): 120-122. doi: 10.32818/reccmi.a5n3a7.

Cite this as: Cantón-De-Seoane J, Flox-Benítez G, Alonso-Martínez B. Tolosa-Hunt Syndrom: an unknow cause of Ophalmoplegia. Rev Esp Casos Clin Med Intern (RECCMI). 2020 (Dec); 5(3): 120-122. doi: 10.32818/reccmi.a5n3a7.

Autor para correspondencia: Juan Cantón-De-Seone. cantonseoane@gmail.com

\section{Palabras clave}

$\triangleright$ Síndrome Tolosa-Hunt

$\triangleright$ Oftalmoplejía

\section{Resumen}

El síndrome de Tolosa-Hunt es una enfermedad rara de causa desconocida que cursa con oftalmoplejía junto parálisis oculomotora. Para su diagnóstico requiere una prueba de imagen o biopsia que objetive inflamación granulomatosa del seno cavernoso. Presentamos una paciente que debuta con clínica de oftalmoplejía e hipoestesia en hemicara izquierda siendo diagnosticada de esta enfermedad mediante resonancia magnética.

\section{Abstract}

Tolosa-Hunt syndrome is a rare illness of unknown origin that develops painful ophthalmoplegia combined with ocular motor nerve palsies. Neuroimaging or biopsy is required to confirm granulomatous swelling in the cavernous sinus. We present a woman with ophthalmoplegia and hypoesthesia on the left side of the head. MRI confirmed diagnosis.

\section{Puntos destacados}

$\triangleright$ El síndrome de Tolosa-Hunt consiste en una inflamación granulomatosa del seno cavernoso.

$\triangleright$ Es un síndrome a tener en cuenta ante clínica de oftalmoplejía y afectación de pares craneales.

$\triangleright$ El tratamiento es corticoides.

\section{Introducción}

El síndrome de Tolosa-Hunt es una enfermedad rara con una incidencia estimada de 1-2 casos por millón. Es de etiología desconocida y afecta de igual forma a cualquier sexo y edad.

La clínica consiste en oftalmoplejía acompañada de paresia de nervios oculomotores además de afectación de otros pares craneales como el trigémino y el facial. Los criterios diagnósticos fueron actualizados en The International Classification of Headache Disorders, 3rd edition (ICHD-3) realizada por la International Headache Society ${ }^{1,2}$.

\section{Caso clínico}

Antecedentes, enfermedad actual y exploración física

Mujer de 40 años de edad que acudió a Urgencias por hipoestesia en hemicara izquierda. Como antecedentes era fumadora de unos 20 cigarrillos al día, hipercolesterolemia controlada mediante tratamiento dietético sin otros factores de riesgo cardiovascular. En estudio por nódulo tiroideo. Su tratamiento habitual era Ranitidina a dosis de un comprimido de 300 miligramos al día.

Un año antes del ingreso, presentó un cuadro de 10 días de evolución de hipoestesia en región malar izquierda, que se extendió posteriormente, a las 3 ramas del trigémino. Fue asociado a patología dentaria y mejoró con tratamiento antibiótico. En los últimos 3 meses refería tos matutina, con expectoración verdosa y rinorrea ocasionalmente purulenta.

El mismo día que acudió a Urgencias presentaba un cuadro de hipoestesia en el ala izquierda de la nariz y en región del pómulo que se extendía posteriormente a toda la cara y se acompañaba con sensación subjetiva de disminución del campo visual izquierdo y alteración de la visión sin clara diplopía. Presentaba, así mismo, dolor con la movilidad del ojo sin otros signos inflamatorios.

A la exploración neurológica presentaba afectación del V, VI y VII par objetivando limitación visual temporal en ojo izquierdo refiriendo dolor con los movimientos, una leve asimetría facial con mínimo borramiento del surco nasogeniano izquierdo sin clara paresia, y una hipoestesia en territorio del trigémino izquierdo con mínima asimetría en la movilidad ocular extrínseca con leve desviación nasal del ojo izquierdo. El resto de la exploración era anodina. El fondo de ojo no presentaba alteraciones. 


\section{Pruebas complementarias}

En la analítica, tanto en la bioquímica como en el hemograma, los valores analíticos estaban dentro de la normalidad. No presentaba elevación de reactantes de fase aguda, la velocidad de sedimentación globular era normal y un estudio de autoinmunidad resultó negativo. Serología de virus de la inmunodeficiencia humana y sífilis negativos. La radiografía de tórax no presentaba alteraciones.

Se solicitó una tomografía axial computarizada (TC) craneal en la que se observó engrosamiento mucoso del seno esfenoidal izquierdo sugerente de patología inflamatoria sin otros hallazgos sugerentes de patología aguda intracraneal (Figura 1).

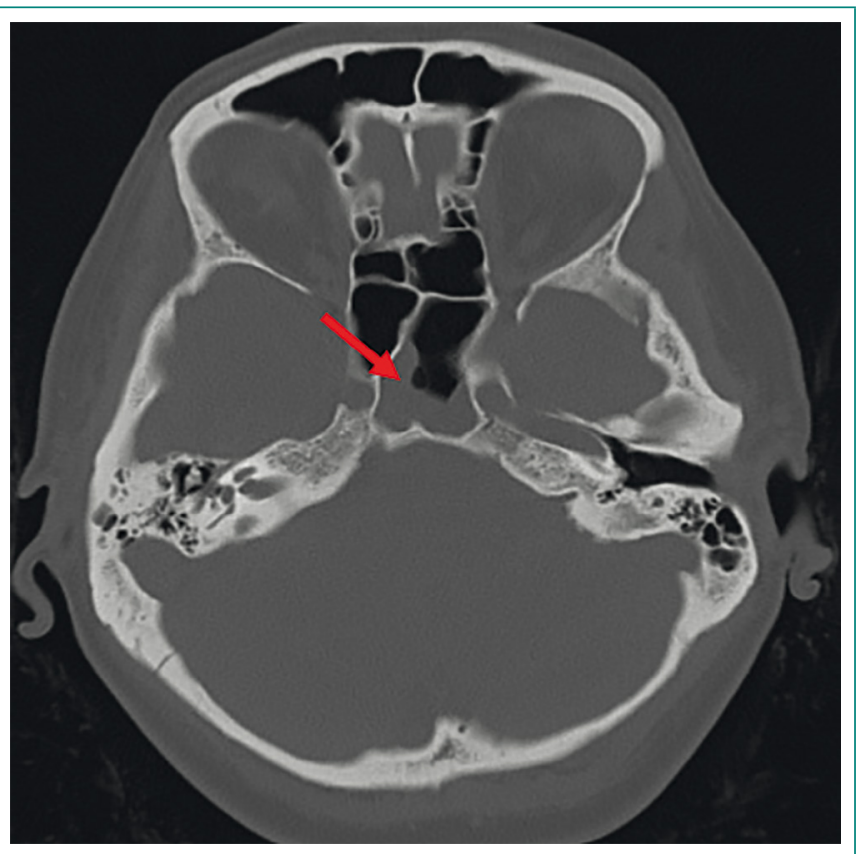

Figura 1. TC corte axial. Se observa engrosamiento mucosos del seno esfenoidal izquierdo.

Se planteó el diagnóstico diferencial de oftalmoplejía junto a hipoestesia facial. Teniendo en cuenta la clínica de meses de evolución con tos, expectoración y rinorrea, sumado a la imagen del TC craneal, una de las posibles causas que se consideró fue la sinusitis con afectación contigua al seno cavernoso.

La causa vascular más frecuente que da lugar a oftalmoplejía es el aneurisma de la arteria carótida intracavernosa. También hay que tener en cuenta otras patologías como: un aneurisma de la arteria cerebral posterior, una neoplasia intracraneal o una metástasis cerebral que invada al seno cavernoso.

La migraña o las cefaleas trigeminales pueden cursar también con oftalmoplejía y paresia de los nervios oculomotores que se puede mantener hasta semanas después del cese de cefalea; sin embargo, la paciente no refiería episodios de cefalea previa. El síndrome de Tolosa-Hunt también cuadraría con la clínica que presentaba la paciente ${ }^{1}$

Finalmente, se solicitó una resonancia magnética (RM) de cráneo y órbitas con contraste. En ella se observó una leve asimetría en senos cavernosos siendo ligeramente mayor el del lado izquierdo, con captación de contraste (Figuras 2 y 3). No se apreciaba extensión anterior a fisura orbitaria superior exoftalmos, ni dilatación de la vena oftálmica superior. Los hallazgos descritos podrían corresponder a patología inflamatoria como el síndrome de Tolosa Hunt (STH).

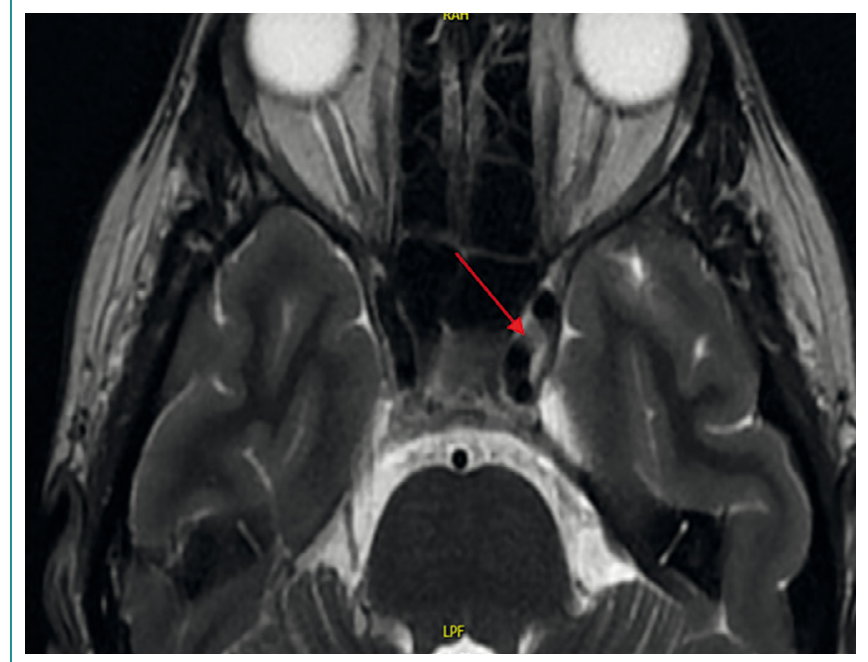

Figura 2. RM corte axial. Se aprecia asimetría de ambos senos cavernosos siendo el mayor izquierdo.

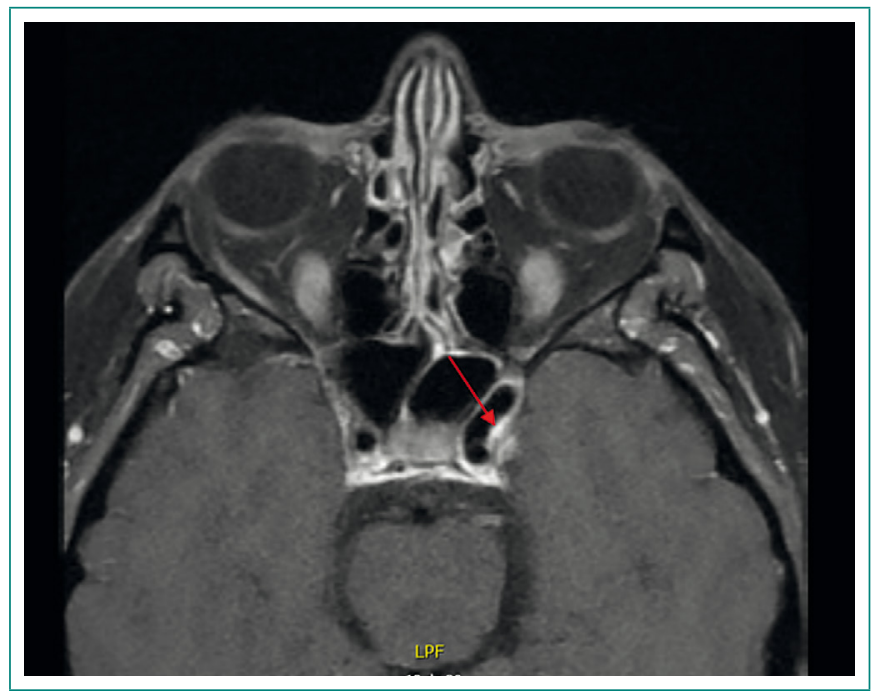

Figura 3. RM corte axial fase T1. Se aprecia que el seno cavernoso izquierdo el ligeramente mayor que el derecho.

\section{Evolución}

Tras los resultados de la RM se inició tratamiento con corticoides a dosis de 80 mg al día con descenso progresivo posterior cada 2 semanas con mejoría clínica posterior.

\section{Diagnóstico}

Síndrome de Tolosa-Hunt.

\section{Discusión y conclusión}

El síndrome de Tolosa-Hunt es una enfermedad rara con una incidencia estimada de 1-2 casos por millón ${ }^{3}$ descrita por primera vez hace más de 50 años por Tolosa y, años después, por Hunt. Afecta por igual a cualquier sexo y no está relacionada con la edad. De etiología desconocida, es causada por un proceso inflamatorio crónico con proliferación de fibroblastos e infiltración del seno cavernoso por linfocitos y células plasmáticas. 
El síntoma principal que afecta a estos pacientes es la oftalmoplejía que puede permanecer durante semanas si no se tarta de forma correcta. El dolor periorbital puede extenderse a zona retroorbital, temporal y frontal. Se acompaña de paresia de cualquiera de los nervios oculomotores que puede aparecer al mismo tiempo que el dolor o transcurridas hasta 2 semanas del inicio del mismo. Puede haber también afectación de la pupila ya sea por afectación de la vía simpática o parasimpática. En ocasiones pueden verse afectados otros pares craneales (trigémino y facial).

El curso clínico es impredecible; puede durar de días a semanas y, hasta en la mitad de los pacientes, recurre al cabo de meses o años de forma ipsilateral contralateral o, raramente, bilateral. Puede persistir una parálisis de un nervio, pero es algo infrecuente ${ }^{1}$.

Los criterios diagnósticos se han ido modificándo a lo largo de los años según los $\operatorname{casos}^{4,5}$. La International Classification of Headache Disorders, 3rd edition (ICHD-3) realizada por la International Headache Society (IHS) refleja los criterios diagnósticos del STH (Tabla 1) ${ }^{1}$

Cefalea orbital o periorbital.

Inflamación granulomatosa del seno cavernoso, fisura orbitaria superior u órbita demostrada por RM o biopsia.

Paresia de uno o más nervios oculomotores ipsilaterales.

Evidencia de que la cefalea es ipsilateral a la inflamación granulomatosa y de que precede a la paresia de los nervios oculomotores dos semanas o se genera con ella.

No cuadra mejor con otro diagnóstico de la ICHD-3.

Tabla 1. Criterios diagnósticos del Síndrome de Tolosa-Hunt.
El tratamiento es con corticoides. Con ellos se objetiva tanto mejoría clínica como radiológica de las lesiones producidas. La cefalea puede llegar a mejorar en 72 horas, mientras que las alteraciones neurológicas requieren hasta 6-8 semanas y las alteraciones radiológicas pueden permanecer hasta meses ${ }^{6}$. La dosis empleada habitualmente es $1 \mathrm{mg} / \mathrm{kg}$ seguido de un descenso progresivo posterior por semana ${ }^{7}$.

\section{Bibliografía}

1. Kline LB, Hoyt WF. The Tolosa-Hunt syndrome. J Neurol Neurosurg Psychiatry. 2001; 71(5): 577-82. doi: 10.1136/jnnp.71.5.577.

2. Headache Classification Committee of the International Headache Society (IHS) The International Classification of Headache Disorders, 3rd edition. Cephalalgia. 2018; 38(1): 1-211. doi:10.1177/0333102417738202.

3. laconetta G, Stella L, Esposito M, Cappabianca P. Tolosa-Hunt syndrome extending in the cerebello-pontine angle. Cephalalgia. 2005; 25(9): 746-50. doi: 10.1111/j.1468-2982.2005.00924.x.

4. Colnaghi S, Versino M, Marchioni E, Pichiecchio A, Bastianello S, Cosi V, et al. ICHD-II diagnostic criteria for Tolosa-Hunt syndrome in idiopathic inflammatory syndromes of the orbit and/or the cavernous sinus. Cephalalgia. 2008; 28(6): 577-84. doi: 10.1111/j.1468-2982.2008.01569.x.

5. La Mantia L, Curone M, Rapoport AM, Bussone G; International Headache Society. Tolosa-Hunt syndrome: critical literature review based on IHS 2004 criteria. Cephalalgia. 2006 Jul;26(7):772-81. doi: 10.1111/j.1468-2982.2006.01115.x.

6. Çakirer S. MRI findings in Tolosa-Hunt syndrome before and after systemic corticosteroid therapy. Eur J Radiol. 2003; 45(2): 83-90. doi: 10.1016/s0720048x(02)00012-8.

7. Kose A, Bozkurt S, Umay ST, Karakulak S, Yilmaz A. A case of headache, double vision and ptosis in emergency department: Tolosa-Hunt syndrome. Turk J Emerg Med. 2017; 18(3): 134-136. doi: 10.1016/j.tjem.2017.10.004. 